\title{
PREVALÊNCIA DE FATORES DE RISCO CARDIOVASCULAR EM PACIENTES FREQUENTADORES DE UM SETOR DE REABILITAÇÃO CARDIOVASCULAR
}

Thais Cabral Almeida ${ }^{1}$, Elaine Aparecida de Oliveira ${ }^{1}$, Gisele Carla Gonçalves dos Santos ${ }^{1}$, Laís Manata Vanzella ${ }^{1}$, Maria Isabela Ramos Haddad ${ }^{1}$, Paula Rapchan dos Santos ${ }^{1}$, Rafaella de Sá Turini Alves ${ }^{1}$, Vitor Eduardo Santos Silva ${ }^{1}$, Anne Kastelianne França da Silva ${ }^{1}$, Luiz Carlos Marques Vanderlei $^{1}$

${ }^{1}$ Universidade Estadual Paulista - UNESP, Curso de Fisioterapia, Presidente Prudente, SP. E-mail: thaiscabral.tc@gmail.com

\section{RESUMO}

A crescente incidência das doenças cardiovasculares (DCV) no último século originou uma busca incessante pelos fatores de risco (FR), pois a prevenção destes permite minimizar a reincidência de eventos cardiovasculares. O objetivo foi analisar a prevalência de FR para DCV em frequentadores de um setor de reabilitação cardíaca. Participaram do estudo 48 voluntários. Foram analisados os FR segundo a ACSM: sexo $\mathrm{x}$ idade, hipertensão arterial, histórico familiar, tabagismo, hipercolesterolemia, obesidade, glicose em jejum e as doenças ou eventos cardiovasculares mais presentes nessa população. Na população estudada o fator de risco mais prevalente é a relação sexo $x$ idade $(89,58 \%)$, seguido de hipertensão arterial $(85,41 \%)$. Existe uma alta prevalência de FR em indivíduos frequentadores de um programa de reabilitação cardiovascular, com isso ressalta-se importância de programas de prevenção primária e secundária de DCV nessa população.

Palavras-chave: fatores de risco, doenças cardiovasculares.

\section{CARDIOVASCULAR RISK FACTORS PREVALENCE IN PATIENTS OF A CARDIOVASCULAR REHABILITATION SECTOR}

\begin{abstract}
The increasing incidence of cardiovascular diseases (CVD) in the last century led to a constant search for risk factors (RF) because the prevention minimizes the recurrence of cardiovascular events. The objective was analyze the prevalence of RF to CVD of goers in a cardiac rehabilitation sector. The study included 48 volunteers. RFs were analyzed according to ACSM: Sex $x$ age, high blood pressure, family history, smoking, hypercholesterolemia, obesity, fasting glucose and diseases or more frequent cardiovascular events in this population. In the population studied the most prevalent risk factor is the rate Sex $x$ age $(89.58 \%)$, followed by hypertension $(85.41 \%)$. There is a high prevalence of RF in goers who regularly attended a cardiac rehabilitation program, therefore it emphasizes the importance of primary prevention programs
\end{abstract}

Keywords: risk factors, cardiovascular disease. 
INTRODUÇÃO

As doenças cardiovasculares (DCV) são atualmente, as principais causas de morbimortalidade em todo mundo ${ }^{1}$ e resultantes da associação entre fatores de risco genéticos e comportamentais ${ }^{2}$.

A crescente incidência das DCV no último século originou uma busca incessante pelos fatores de risco $(F R)^{3}$ que predispõe à sua ocorrência. Ainda que FR como genética e idade tenham grande importância nesta evolução, boa parte dos outros FR podem ser influenciados por mudanças no estilo de vida ${ }^{3}$.

Segundo a ACSM (American College of Sports Medicine), entre os FR considerados de maior importância para o desenvolvimento de DCV destacam-se o histórico familiar, tabagismo, hipertensão arterial, hipercolesterolemia, glicemia de jejum alterada, obesidade e sedentarismo ${ }^{4}$. Ainda, quando agregados, os comportamentos de risco como o sedentarismo, obesidade e tabagismo, aumentam a chance de o indivíduo apresentar $\mathrm{FR}^{5}$, sugerindo que a combinação destes fatores para o desenvolvimento de DCV é maior do que se considerados isoladamente ${ }^{6}$.

A prevenção dos fatores de riscos para DCV, especialmente os de natureza comportamental, permite implementar ações preventivas com maior custo-efetividade, e, portanto minimizar a reincidência de eventos cardiovasculares ${ }^{1}$. Com isso, o setor de saúde e as políticas públicas envolvidas no combate aos FR para DCV utilizam como estratégia para reduzir essa prevalência, a prevenção primária de FR por meio de informações para conscientização da população a cerca dos FR existentes e como o indivíduo pode atuar para reduzir a sua incidência ${ }^{1,3}$ Além disso, o conhecimento a respeito das DCV e os fatores que levam o seu desenvolvimento, possibilitam aos pacientes entenderem a importância da prevenção dos FR e o impacto positivo na sua saúde.

Diante disso e da importância do tema na saúde pública, torna-se necessário investigar os FR presente em uma população que já apresenta DCV, com o objetivo de promover um programa de prevenção que atue nos principais FR que afetam essa população.

Sendo assim, o objetivo deste estudo foi analisar a prevalência de FR para DCV em uma população frequentadora de um centro de reabilitação cardiovascular.

\section{METODOLOGIA}

Para a realização desse trabalho foram analisados dados de 48 voluntários, que frequentam o Setor de Reabilitação Cardiovascular do Centro de Atendimentos em Fisioterapia e Reabilitação (CEAFiR) da Faculdade de Ciências e Tecnologia de Presidente Prudente (FCT/UNESP). 
Para a caracterização dos dados foram coletados dados das fichas de controle diário e dos prontuários dos participantes do programa. A idade foi colhida em anos completos. E quanto ao gênero, a população foi classificada como masculino e feminino. Os procedimentos utilizados nesse estudo foram aprovados pelo Comitê de Ética e Pesquisa da Instituição (Proc. no do CAAE: 35525714.9.0000.5402).

Foram analisados os FR segundo a ACSM: sexo $x$ idade (homens $>45$ anos e mulheres $>55$ anos), hipertensão arterial (PAS $\geq 140 \mathrm{mmHg}$ ou $\mathrm{PAD} \geq 90 \mathrm{mmHg}$ em repouso, controlada por medicamento), histórico familiar, tabagismo, hipercolesterolemia (diagnosticada LDL $>130 \mathrm{mg} / \mathrm{dL}$ ou colesterol sérico total $>200 \mathrm{mg} / \mathrm{dL}$ e $\mathrm{HDL}<40 \mathrm{mg} / \mathrm{dL}$ ), obesidade (IMC $>30 \mathrm{Kg} / \mathrm{m}^{2}$ ) e glicose em jejum (> 100mg/dl) ${ }^{7}$. Os dados foram coletados através de prontuários dos pacientes para investigação de sexo $\mathrm{x}$ idade, histórico familiar, tabagismo e com base nos medicamentos utilizados foi verificado o uso de anti-hipertensivos; a hipercolesterolemia e a glicose de jejum também foram verificadas em exame com data não superior a um ano e os pacientes tiveram mensurado o peso e a estatura para cálculo do $\mathrm{IMC}\left(\mathrm{kg} / \mathrm{m}^{2}\right)$

Além disso, foram investigadas as doenças ou eventos cardiovasculares mais presentes nessa população, mediante a comprovação de exame e/ou laudo médico.

Os dados obtidos foram organizados em planilha computacional e estatística descritiva foi utilizada para sua análise. Os resultados foram apresentados em números absolutos e percentuais.

\section{RESULTADOS}

Nossos resultados apontam que na população estudada, 60,41\% pertenciam ao sexo masculino (29 pacientes) e 39,58 \% ao sexo feminino (19 pacientes) e a média de idade observada foi de $67,14 \pm 11,83$ anos. Os diagnósticos principais mais incidentes encontrados nessa população foi o de insuficiência coronariana $(47,91 \%)$, seguido de infarto agudo do miocárdio $(16,66 \%)$ e miocardiopatia dilatada (8,33\%), como observado na tabela 1. 
Tabela 1. Caracterização da população estudada e diagnósticos mais frequentes $(n=48)$.

\begin{tabular}{lc}
\hline \multicolumn{1}{c}{ Características } & Valores \\
\hline Gênero M/F & $29(60,41 \%) / 19(39,58 \%)$ \\
Idade anos & $67,14 \pm 11,83$ \\
Diagnóstico Principal & \\
Insuficiência coronariana & $22(45,83 \%)$ \\
Infarto agudo do miocárdio & $8(16,66 \%)$ \\
Miocardiopatia dilatada & $4(8,33 \%)$ \\
Outros diagnósticos cardiovasculares & $3(6,25 \%)$ \\
Fatores de riscos cardiovasculares & $11(22,91 \%)$ \\
\hline
\end{tabular}

Valores expressos como média \pm desvio-padrão ou em números absolutos e percentuais.

Legenda: $M=$ masculino; $F=$ feminino

A tabela 2 mostra a prevalência dos fatores de risco Sexo X idade, hipertensão arterial, histórico familiar, hipercolesterolemia, obesidade, tabagismo e glicose de jejum alterada, dos frequentadores do setor de reabilitação cardiovascular, de acordo com os critérios da ACSM $^{7}$. Podemos observar que houve prevalência dos fatores de risco: Sexo x idade $(89,58 \%)$, seguido de Hipertensão arterial (85,41\%), Histórico familiar (70,83\%), Hipercolesterolemia (64,58\%), Obesidade (31,25\%), Tabagismo (6,25\%) e Glicose jejum alterada $(4,16 \%)$.

Tabela 2. Prevalência dos fatores de risco segundo critérios da ACSM de indivíduos frequentadores do setor de reabilitação cardiovascular.

\begin{tabular}{lcc}
\hline \hline Fatores de risco & $\boldsymbol{n}$ & $\%$ \\
\hline Sexo x idade & 43 & 89,58 \\
Hipertensão arterial & 41 & 85,41 \\
Histórico familiar & 34 & 70,83 \\
Hipercolesterolemia & 31 & 64,58 \\
Obesidade & 15 & 31,25 \\
Tabagismo & 3 & 6,25 \\
Glicose jejum alterada & 2 & 4,16 \\
\hline \hline
\end{tabular}

$\mathrm{n}$ : número absoluto. 


\section{DISCUSSÃO}

Os nossos resultados apontam alta prevalência de FR em frequentadores de um programa de reabilitação cardíaca. Além disso, observamos que dos 48 pacientes analisados, $60,41 \%$ são do sexo masculino, demonstrando predomínio desse gênero neste programa de reabilitação cardiovascular, semelhante ao que foi observado por Lopes et $\mathrm{al}^{8}$, ao apontarem que $53,41 \%$ dos pacientes frequentadores de programas ambulatoriais de exercícios físicos eram do sexo masculino.

Em relação aos FR, observamos que a hipertensão arterial (HA) foi um dos fatores mais prevalentes, assim como a relação sexo $\mathrm{x}$ idade. Para Oliveira et al. $^{9}$ a prevalência de HA em pessoas acima de 60 anos está acima de $50 \%$, o que foi condizente com nosso trabalho $(85,41 \%)$. Ainda, a prevalência de HA também foi observada por Pansani et $\mathrm{al}^{10}$, ao observarem prevalência de 59,92\% de idosas hipertensas. De acordo com os autores essa elevada prevalência pode ser parcialmente explicada pela idade avançada, uma vez que no estudo foi observada associação significante entre ambos ${ }^{10}$. Outros autores também relataram aumento proporcional da pressão arterial com a idade, o que pode ser explicado pela diminuição da elasticidade arterial com o envelhecimento, induzindo ao aumento da resistência periférica ${ }^{11,12}$.

Outro fator que pode interferir na prevalência de hipertensos é a obesidade; diversos estudos evidenciaram que está associada a níveis pressóricos mais elevados e que o ganho de peso que acompanha o envelhecimento é um importante preditor para o desenvolvimento de $H A^{13}$. Em nosso estudo, 31,25 \% dos pacientes eram obesos, sendo $60 \%$ homens e $40 \%$ mulheres. Além disso, a prevalência de indivíduos com colesterol elevado foi de $64,58 \%$. Diversos estudos apontam que níveis elevados de colesterol estão associados a outros FR, como a obesidade, e também a hipertensão arterial, uma vez que com o aumento de colesterol os indivíduos ficam mais propensos a aterosclerose e como consequência a eventos cardiovasculares ${ }^{14}$.

Quanto à hereditariedade, 70,83\% da população analisada apresentou histórico familiar de DCV. Mesmo se tratando de um FR não modificável, a alta prevalência deste FR indica que indivíduos que apresentam histórico familiar de DCV podem ser beneficiados por programas de prevenção de FR, uma vez que podem estar mais propensos ao desenvolvimento de DCV, devido a hereditariedade ${ }^{4}$.

Na população estudada a prevalência de tabagismo e de glicemia alterada no jejum foi baixa, $6,25 \%$ e $4,16 \%$ respectivamente, entretanto é importante ressaltar, mesmo com esse fato de baixa incidência, a informação sobre esses fatores de risco são essenciais, pois eles estão 
diretamente ligados a DCV e sabe-se que a somatória de fatores de risco aumenta ainda mais a chance de ocorrer eventos cardiovasculares ${ }^{6}$.

Portanto, o presente estudo mostrou a alta prevalência de FR em indivíduos que participam de um programa de reabilitação cardiovascular. Visto que essa população já possui algum tipo de DCV, essa alta prevalência pode deixar o individuo mais susceptível à ocorrência de outro evento cardiovascular, agravando a sua saúde.

Diante disso, programas de caráter preventivo são de extrema importância, uma vez que atuam conscientizando e mostrando à população a importância de um bom controle de pressão arterial, colesterol, glicemia, cessação do tabagismo, prática de atividade física e reeducação alimentar, a fim de diminuir a prevalência e evitar complicações futuras.

\section{CONCLUSÃO}

Os resultados permitem concluir que, existe uma alta prevalência de FR em indivíduos com DCV frequentadores de um programa de reabilitação cardiovascular. Com isso, ressalta-se a importância de programas de prevenção primária e secundária de DCV e da presença de orientações a respeito das mudanças no estilo de vida dessa população.

\section{REFERÊNCIAS}

1. Ribeiro AG, Cotta RMM, Ribeiro SMR. A promoção da saúde e a prevenção integrada dos fatores de risco para doenças cardiovasculares. Cien e Saude Colet. 2012;17(1):7-17. http://dx.doi.org/10.1590/S1413-81232012000100002

2. Oliveira MAM, Fagundes RLM, Moreira EAM, Trindade EBSM, Carvalho T. Relação de indicadores antropométricas com fatores de risco para doença cardiovascular. Arq Bras Cardiol. 2010;94(4):478-85. http://dx.doi.org/10.1590/S0066-782X2010005000012

3. Ferreira AIS, Ferreira G. Prevalência de mortalidade por doenças cardiovasculares em uma cidade do Sul de Minas Gerais nos anos de 1999 a 2008. Rev Cien em Saude. 2012;2(2):1-10.

4. ACSM. Guidelines for exercise testing and prescription. ACSM'S Health \& Fitness Journal. 2013;17(2):16-20.

5. Bernado AFB, Fernandes RA, Silva AKF, Valenti VE, Pastre CM, Vanderlei LCM. Influence of risk behavior aggregation in different categories of physical activity on the occurrence of cardiovascular risk factors. Int Arch Med. 2013;6:26. http://dx.doi.org/10.1186/1755-7682-6-26

6. Pimenta HB, Caldeira AP. Fatores de risco cardiovascular do Escore de Framingham entre hipertensos assistidos por equipes de saúde da família. . Cien e Saude Colet. 2014;19(6):1731-39. http://dx.doi.org/10.1590/1413-81232014196.20092013 
7. ACSM. Triagem de Saúde e Estratificação dos Riscos (Cap. 2). Princípios Gerais da Prescrição do Exercício (Cap. 7) In: Diretrizes do ACMS para os testes de esforço e sua prescrição. 6a ed., 2000.

8. Lopes PP, Vanderlei LCM, Tarumoto MH. Perfil dos indivíduos frequentadores de programas ambulatoriais de exercícios físicos para pacientes cardíacos da cidade de Presidente Prudente (SP). Rev Soc Cardiol Estado de SP. 2005;15(3):19-25.

9. Oliveira JJ, Silva SR. O idoso com hipertensão arterial. RBM Rev Bras Med. 1999;56(7):56480.

10. Pansani AP, Anequini IP, Vanderlei LCM, Tarumoto MH. Prevalência de fatores de risco para doenças coronarianas em idosas frequentadoras de um programa "Universidade aberta à terceira idade". Arq Cienc Saúde. 2005;12(1):27-31.

11. Arquino EMLL, Magalhaes LBNC, Araújo MJ, Almeida MCC, Leto JP. Hipertensão arterial em trabalhadores de enfermagem: padrão de ocorrência, diagnóstico e tratamento. Arq Bras Cardiol. 2001;76(3):197-208.

12. Oigam W. Adaptação dos vasos arteriais e do coração à hipertensão arterial. Sinergia. $2001 ; 2(2): 2-8$.

13. Brandão AP, Brandão AA, Magalhães MEC, Pozzan R. Epidemiologia da hipertensão arterial. Rev Soc Cardiol Estado SP. 2003;13(1):7-19.

14. Carneiro G, Faria AN, Filho FR, Guimarães A, Lerário D, Ferreira SRG et al. Influencia da distribuição da gordura corporal sobre a prevalência de hipertensão arterial e outros fatores de risco cardiovascular em indivíduos obesos. Rev Assoc Med Bras. 2003;49(3):306-11. http://dx.doi.org/10.1590/S0104-42302003000300036 